cemoti $\begin{aligned} & \text { Cahiers d'études sur la Méditerranée } \\ & \text { orientale et le monde turco-iranien }\end{aligned}$

19 | 1995

Laïcité(s) en France et en Turquie

\title{
Demande de l'Islam en banlieue : un défi à la citoyenneté
}

Jocelyne CESARI

\section{(2) OpenEdition \\ Journals}

Édition électronique

URL : http://journals.openedition.org/cemoti/1695

DOI : $10.4000 /$ cemoti. 1695

ISSN : 1777-5396

Éditeur

AFEMOTI

Édition imprimée

Date de publication : 1 janvier 1995

ISSN : 0764-9878

Référence électronique

Jocelyne CESARI, «Demande de l'Islam en banlieue : un défi à la citoyenneté », Cahiers d'études sur la Méditerranée orientale et le monde turco-iranien [En ligne], 19 | 1995, mis en ligne le 14 mai 2006,

consulté le 07 septembre 2020. URL : http://journals.openedition.org/cemoti/1695 ; DOI : https:// doi.org/10.4000/cemoti.1695

Ce document a été généré automatiquement le 7 septembre 2020

Tous droits réservés 


\title{
Demande de l'Islam en banlieue : un défi à la citoyenneté
}

\author{
Jocelyne CESARI
}

En l'espace d'une dizaine d'années, la demande d'islam s'est affirmée comme un élément majeur du processus de sédentarisation des populations immigrées originaires du Maghreb, de l'Afrique subsaharienne et de la Turquie. Les signes de l'appartenance islamique se sont multipliés dans l'espace public : salles de prière, boucheries halal, port du foulard, carrés musulmans dans les cimetières etc. L'interprétation de cette dimension islamique de la migration va susciter quatre principaux types de représentations, non exclusives l'une de l'autre et qui contribuent à façonner les prises de position et les orientations concernant ce qu'il est désormais convenu d'appeler "la deuxième religion de France". La première de ces représentations consiste à considérer les manifestations de l'appartenance islamique comme les indices d'une réislamisation alors que dans la plupart des cas elles sont le révélateur d'un changement d'attitude par rapport à la société française. L'émergence d'un islam public va également être interprétée comme la conséquence de manipulations extérieures pouvant s'accompagner d'une déstabilisation politique car elle se situe au moment où cette religion surgit sur la scène internationale et devient un élément de la lutte politique et de la mobilisation des masses dans le monde arabo-islamique. Ce plaquage de la situation internationale sur le contexte français a conduit à occulter l'innovation sociale que constitue la transplantation de l'islam pour les musulmans dans la mesure où l'exil engendre inéluctablement une modification dans les systèmes de signification. Le fait de décoder toute manifestation islamique comme l'expression d'un fondamentalisme est également très répandu. Il se produit alors un effet de totalisation qui rend impensable la prise en compte des libertés, des accommodements et des compromis que tout musulman exerce par rapport à la Loi Révélée à l'instar de n'importe quel autre croyant. Enfin, toutes ces représentations se trouvent en quelque sorte subsumées dans l'idée largement partagée selon laquelle l'islam est incompatible avec les normes et valeurs de la société française synthétisées dans la laïcité. Il en résulte un processus d'illégitimation de toute forme d'appartenance islamique que l'on peut interpréter comme l'une des séquelles de l'histoire coloniale ${ }^{1}$. 
2 C'est dans ce contexte, qu'il faut reposer la question du rapport des musulmans à la société française, en évitant l'écueil du contre-discours de légitimation face au processus d'illégitimation dont ils sont l'objet et qui nourrit les interprétations qui viennent d'être évoquées. Pour celà il faut partir non pas des préjugés qui les concernent mais des logiques propres qui sous-tendent leurs comportements et leurs actions collectives.

3 Les modalités d'appartenance à l'islam se distinguent en fonction des parcours migratoires, des histoires nationales des groupes de migrants mais aussi de l'âge et du sexe et ne prennent de sens en France qu'en tenant compte de toutes ces dimensions qui rendent impossible la préhension d'une communauté musulmane, même si certains leaders religieux ont tout intérêt à la promotion et à la légitimation de cet artefact. Ces modalités plurielles d'identification à l'Islam s'accompagnent de rapports différents à la citoyenneté et à la laïcité. En ce qui concerne les populations musulmanes, originaires du Maghreb, deux modalités principales de vivre son appartenance religieuse émergent ${ }^{2}$ : soit le référent islamique fonctionne comme principal organisateur du sens des conduites et des comportements en société, soit il n'est envisagé que dans sa dimension culturelle sans véritable implication sur les comportements en société. Cette distinction recoupe des modes de socialisation et donc des générations mais aussi des milieux sociaux différents.

L'ISLAM COMME MODE DE VIE

4 Depuis une décennie, la volonté d'apparaître comme musulman dans la société d'accueil brise le repli qui a longtemps caractérisé les primo-migrants maghrébins par rapport à l'espace public français. En effet, pour ceux-ci l'identification à la nation d'origine neutralisait toute action d'inclusion dans la société d'accueil et conduisait à des actes de dénégation envers les conséquences de la migration, qu'il s'agisse de retarder le plus possible le regroupement familial ou encore de limiter les engagements politiques ou syndicaux ${ }^{3}$. Ceci s'explique par le fait que la migration était un projet de mobilité et de déplacement en vue d'une accumulation de capital devant être réinvesti dans le pays d'origine. Or, l'échec de ce projet, patent au début de la décennie 1980, va contribuer à modifier la relation de ces immigrés à la société française et conduire à un investissement symbolique dans l'islam qui apparaît alors comme un moyen de recomposer l'unité perdue et de compenser les conséquences sociales de l'impossibilité $\mathrm{du}$ retour. Tout se passe désormais comme si l'identification à l'islam se muait en identification sociale permettant de résister à l'environnement extérieur et fonctionnait comme une nationalité de substitution et de compensation. Ce mode d'identification permet de définir les contours d'un premier groupe d'individus relativement homogène en fonction de l'âge, du sexe et de la durée d'installation en France. Il s'agit en général, d'hommes âgés de plus trente-cinq ans, mariés, pères de famille, venus en France depuis plus d'une décennie dans le cadre de la migration de travail et dont les enfants sont nés ou ont été scolarisés dans la société d'accueil.

5 On peut également observer cette place centrale du référent islamique au sein d'une fraction minoritaire de la classe d'âge 18-24 ans. Ce sont en général des hommes nés ou scolarisés en France qui vont effectuer non pas un retour mais une découverte de l'islam. Ce passage à la pratique apparaît comme une alternative crédible dans la définition de soi après quelques années d'errance où le chômage, la petite délinquance voire la drogue et l'alcool ont souvent été leur lot quotidien. Cette appartenance "revisitée" permet de redonner sens et cohérence à l'existence d'individus en voie de 
marginalité, situés à la périphérie du système social et politique. Il convient de préciser que cette valorisation ne passe pas par le milieu familial mais plutôt par des personnes extérieures qui renvoient une image très positive de la religion : français convertis à l'islam et surtout étudiants provenant du Maghreb plus lettrés que les parents et qui vont faire acte de prosélytisme auprès de ces nouvelles générations. Il faut enfin souligner que cette islamisation des jeunes reste à ce jour marginale, la plupart étant caractérisés par un mode de relation à l'islam plus sécularisés ${ }^{4}$.

6 Pour ces deux composantes de la population musulmane, la mise en oeuvre des prescriptions islamiques va constituer un moyen d'éviter l'aliénation et d'échapper aux modèles allogènes de la société d'accueil. Une "totalisation existentielle" dans et par l'islam s'opère, c'est-à-dire un processus d'unification du groupe par les pratiques et le sens conféré au référent islamique. Le fait de se considérer comme musulman est alors un moyen de reconquête de la dignité qui fait que l'on n'est pas totalement perdu dans l'exil ou dans l'errance. Etre musulman consiste à interpréter sa propre existence et son environnement immédiat en fonction de deux dimensions: l'eschatologie et le sacré.

7 La première de ces dimensions renvoie à une division entre monde "d'en haut" et monde "d'en bas". La vie d'en bas est envisagée en fonction du salut éternel à obtenir dans l'au-delà, ce qui va conduire à un désintérêt très marqué pour toute activité qui n'est pas reliée à cet impératif. La seconde dimension renvoie à la polarité entre le pur et l'impur, (le licite et l'illicite) haram/halal qui permet de classer les actes permis et interdits. Cette polarité se concrétise dans le maintien des pratiques alimentaires issus des interdits religieux ainsi que dans la séparation homme/femme difficile à instaurer dans la société française. C'est pourquoi, l'espace des salles de prière, créées et investies par des hommes permet de reconstituer cette bipolarité ignorée ou méprisée dans les espaces de travail ou d'habitation. De manière générale, ces musulmans tentent de reconstituer un "entre-nous" en opposition au monde extérieur considéré comme lieu de souillure et de dépravation. Dans la reconstitution de cet "entre-nous" fondée sur l'opposition haram/halal, la condition de la femme tient une place significative que ce soit à propos de son vêtement ou de son statut dans le couple ou le monde du travail.

8 Cette centralité de l'islam dans le sens que ces individus confèrent à leur vie en France s'accompagne le plus souvent d'une visibilité dans les manifestations de l'appartenance islamique.

De l'invisible au visible

$9 \mathrm{Si}$, dans l'islam "transplanté", les primo-migrants n'ont jamais véritablement abandonné les pratiques individuelles comme les prières, la saddaka ou le haj, désormais ils affichent dans l'espace public la dimension collective du culte par la multiplication des salles de prière, des boucheries "halal" ou la demande de carrés musulmans dans les cimetières. Cela consacre une évolution dans leurs rapports à l'environnement. En effet, jusqu'au début de la décennie 1970, aucun signe cultuel n'apparaissait dans les espaces urbains : la dimension collective de l'islam était refoulée dans l'espace intime des demeures, des foyers, des hôtels garnis ou des arrièreboutiques. Cette attitude de repli s'explique par le fait que pour la majorité d'entre eux, la société d'accueil était pas envisagée comme un lieu d'enracinement mais comme un lieu de passage, ce qui permettait aussi de faire quelques entorses au respect des prescriptions rituelles 5 . 
10 Mais avec la recomposition des familles sur le territoire français s'accompagnant de la naissance d'enfants ou de leur scolarisation en France, il n'est plus possible pour le croyant de vivre dans l'irrégularité par rapport à Dieu, même si l'idée de retour n'est pas abandonnée. Il va en résulter à partir de la décennie 1980, une croissance exponentielle des salles de prière dans l'ensemble des lieux de résidence ${ }^{6}$. Cette émergence de l'islam dans les banlieues va être interprétée par les pouvoirs publics comme le signe d'une non adaptation alors qu'elle est au contraire l'indice d'une acceptation de l'environnement. Elle va d'ailleurs contraindre les fidèles à entrer en négociation avec les responsables des sociétés HLM et du pouvoir municipal et à créer des associations loi de 1901 afin d'apparaitre comme des interlocuteurs face aux pouvoirs publics, ce passage à la structure associative ayant été favorisée par la loi du 9 octobre 1981 qui a fait entrer les associations créées par les étrangers dans le régime général des associations loi de 1901. Il convient de souligner que cette création d'associations demeure dans la plupart des cas formelle, les lieux islamiques étant gérés à partir du réseau de relations personnelles. Ils fonctionnent davantage comme des lieux communautaires: le régime juridique n'est qu'un outil pour acquérir une reconnaissance auprès des institutions, l'organisation interne étant fondée sur les réseaux de solidarité régionale et nationale.

11 Les conditions de création de ces salles de prière mettent en évidence non seulement une logique d'adaptation à l'environnement immédiat mais aussi une autonomie des fidèles par rapport aux institutions des pays d'origine. En effet, l'organisation des activités religieuses est d'abord le fait des pratiquants eux-mêmes, à partir de réseaux de solidarité recréées dans les quartiers en fonction d'affinités régionales, nationales et ethniques. Les institutions étatiques ou associatives des pays d'origine n'interviennent que dans un second temps lorsque les fidèles ont besoin d'un financement complémentaire ou d'une aide matérielle. Ceci n'empêche pas que ces institutions tentent de contrôler ces lieux par divers procédés : envoi d'imams temporaires ou définitifs, mise à disposition de formateurs de langue arabe pour dispenser des cours aux enfants etc.. Les pratiquants peuvent également solliciter des aides financières auprès d'autres pays musulmans : principalement l'Arabie Saoudite par l'intermédiaire de la Ligue islamique mondiale ou encore les Emirats. Mais, contrairement aux idées reçues, cette aide financière ne s'accompagne par d'une main mise sur les lieux de culte par ces instances donatrices, tout simplement parce qu'elles n'en ont pas les moyens mai aussi parce que les fidèles font preuve d'une grande résistance face à toute tentative d'ingérence extérieure ${ }^{7}$.

12 En fait, ces lieux de prière mettent en évidence la constitution de groupes ethniconationaux à l'échelle des quartiers. La preuve en est que sur une même unité de résidence, il n'est pas rare de trouver un lieu de culte pour "Maghrébins" jouxtant un lieu de culte pour "Africains" ou "Turcs". Ces communautés ethnico-nationales de quartier remplissent plusieurs fonctions :

13 - Elles sont un moyen symbolique de recomposition d'une unité perdue et d'un projet d'accumulation échoué.

14 - Elles remettent de l'ordre dans les sexes. Dans la mosquée, l'homme adulte recherche une re-légitimation de l'autorité masculine paternelle et maritale qu'il est en train de perdre : le chômage mine le pouvoir qui lui vient de l'apport monétaire, les nouvelles générations se réfèrent à d'autres modèles d'autorité, les femmes et surtout les filles exigent de nouveaux rôles à l'intérieur et à l'extérieur de la famille. 
15 - Enfin, elles apparaissent comme un moyen de résistance au processus général de déculturation. Elle nourrissent ainsi l'opposition intérieur/extérieur qui se décline autour des couples suivants: souillure/pureté, incertitude/certitude, mais aussi société/communauté.

Par ces différentes fonctions, ces communautés "ethnico-nationales-religieuses" dessinent les frontières d'une zone de repli et de confirmation imaginaire de la position de marginalité à partir de laquelle ces musulmans s'inscrivent dans la société d'accueil. Ils apparaissent ainsi dans une attitude de retrait par rapport à la société française, ce qui contribue à façonner leur sentiment d'appartenance et d'engagement par rapport à la collectivité locale ou nationale. En terme d'appartenance, ils sont en effet caractérisés par une identification prioritaire à des communautés de quartier créées à partir du lien ethnico-religieux tandis que leur engagement dans la cité oscille entre un sens de l'obligation envers l'Etat comme permettant l'exercice de son activité spécifique et donc dispensateur de droits spécifiques et une certaine autonomie, ce terme renvoyant à l'attitude de personnes liées à l'Etat pour leur subsistance mais fondamentalement non engagés dans les affaires et les problèmes publics. Cette allégeance à des groupes restreints cumulée à une adhésion minimale à l'ordre commun dessine les contours d'une citoyenneté passive voire négative lorsque l'Etat n'est envisagé que dans sa fonction d'allocations de ressources et de droits ${ }^{8}$.

17 Cette "totalisation existentielle" autour du référent islam ainsi que la visibilité croissante de cette appartenance amène à poser l'éventualité du réaménagement entre domaine public et domaine privé que ces musulmans sont prêts à négocier dans une société non musulmane.

Vers une privatisation de l'islam?

18 Contrairement aux lieux communs les mieux partagés, la question n'est pas de savoir si ces musulmans sont dans l'impossibilité de séparer le politique du religieux. Certes, l'articulation de l'autorité spirituelle et du pouvoir politique a été et reste l'un des points les plus lancinants et les plus débattus du monde musulman. L'existence d'une sphère politique différenciée et indépendante de l'ordre religieux est impensée dans les traditions islamiques. C'est une des difficultés majeures à laquelle se heurte aujourd'hui la légitimité des Etats construits après la décolonisation dans le Dar-Al-Islam. Mais cela ne signifie pas pour autant que dans les faits les deux instances aient été confondues. C'est ainsi qu'un aménagement du temporel a pu trouver place à l'intérieur des normes divines positives'.

19 Comment alors les populations musulmanes vivent-elles au sein d'un Etat français dont la légitimité n'obéit pas à des règles transcendantes et dont l'enjeu réside dans la mise en oeuvre de compromis sociaux provisoirement stables? Il faut préciser que dans l'abondante jurisprudence islamique, l'éventualité de l'existence de minorités musulmanes et de leurs rapports à un pouvoir non musulman n'a pas été prise en considération pour des raisons historiques tenant au fait que la pensée juridique s'est construite et fixée au moment de la domination musulmane, c'est-à-dire du temps des Empires umayyade et abasside ${ }^{10}$. Mais, l'allégeance à une autorité non musulmane n'est pas un enjeu majeur dans la condition des musulmans de France puisque après tout les pouvoirs politiques mis en place après les indépendances dans les pays d'origine sont en grande partie sécularisés. En revanche, la question du réaménagement éventuel des domaines respectifs du privé et du public que ces musulmans sont prêts à négocier (ou pas) face à un Etat-nation laïque mérite d'être posée car elle réfère à la revendication 
éventuelle de l'application des règles de statut personnel. En d'autres termes, est-ce qu'une totalisation existentielle dans l'islam conduit à l'islam comme "revendication totalisante"?

20 Il est possible de distinguer plusieurs niveaux d'appréhension de cette question de la part de ceux qui envisagent l'islam comme un mode de vie. A un premier niveau, le compromis apparaît comme nécessaire car la France n'est pas un pays musulman et en même temps ce compromis est acceptable car la possibilité de respecter les cinq piliers est bon an mal an assurée. Tous les témoignages recueillis attestent de cette conception de la France comme espace pluriel dans lequel la pratique de l'islam est possible. Les critiques portent sur les obstacles rencontrés dans la mise en oeuvre de cette pratique et qui repèrent davantage à des blocages de mentalité polutôt qu'à des blocages juridiques. Dès lors semble se dessiner un paradoxe entre le processus de "totalisation existentielle" par l'islam et cette acceptation d'une approche réduite au cinq piliers. Ceci renvoie à un second niveau qui fait que la France est perçue notamment parmi les plus jeunes de ces pratiquants comme une terre de Prédication. Dans cette perspective, revendiquer les règles de Statut personnel dans le court terme n'a pas de sens dans la mesure où avec la force de persuasion des "vrais croyants", celles-ci s'imposeront d'elles-mêmes, c'est-à-dire lorsque bon nombre de Français seront devenus musulmans, suffisamment du moins pour que la France soit considérée comme faisant partie du Dar-al-Islam.

21 Il convient de préciser qu'une telle conception ne répond pas aux attentes de la majorité des nouvelles générations nées ou scolarisées en France. Pour celles-ci, la référence islamique est positionnée dans la sphère personnelle voire intime, sans véritable conséquence sur les comportements en société. Ceci révèle une relation éminemment moderne à l'islam liée à leur acculturation et qui va les conduire à marquer leur individualité et à se placer comme médiateur entre le contenu du code et son application. Plus les personnes ont acquis du capital culturel en France et plus cette attitude est affirmée.

Bien que la revendication du Code de Statut personnel ne soit pas pour l'instant à l'ordre du jour ${ }^{11}$, le rapport au institutions politiques des musulmans qui font de l'Islam un mode de vie révèle d'une part, un manque d'expérience de la condition minoritaire, d'autre part un manque d'intelligibilité de la laïcité.

L'ORGANISATION DE L'ISLAM DE FRANCE : UN DEFI A LA LAICITE?

23 Comme nous l'avons souligné, les associations islamiques créées sous le régime de la loi de 1901 par les "pratiquants installés" correspondent avant tout à la recomposition de liens communautaires et de sociabilité à l'échelle d'un quartier. Or ces associations vont devenir le lieu d'enjeux qui dépassent leurs promoteurs.

L'enjeu de la représentativité

24 En effet, la sédentarisation de l'islam s'accompagne de demandes religieuses de tous ordres (consommation de viande halal, enseignement coranique, gestion de la mort etc.) dont la satisfaction dépasse les compétences des petits groupes ethnico-nationaux précédemment décrits. En effet, enracinés dans une aire géographique délimitée, bien souvent ils ne détiennent pas de légitimités et de ressources symboliques suffisantes pour être les acteurs du passage à des revendications d'ordre général concernant le statut de l'islam. Il en résulte des rivalités entre divers responsables d'associations de quartier pour apparaître comme des interlocuteurs légitimes dans l'espace local en même temps qu'émergent des acteurs dont les intérêts sont extérieurs aux groupes de 
"pratiquants installés". Ainsi, l'enjeu de l'organisation d'une instance représentative des musulmans de France, peu intelligible par les pratiquants est souvent porté par des acteurs inscrits dans des réseaux transnationaux passant par les pays d'origine. C'est pourquoi, des courants divers et souvent antagonistes traversent ces associations liés soit aux intérêts des Etats d'origine soit à des mouvements d'opposition aux régimes politiques d'outre-Méditerranée. La division et l'hétérogénéité de ce mouvement associatif conduisent alors les municipalités à apparaître comme des acteurs centraux dans le processus d'organisation de la minorité musulmane au niveau local dans le cas notamment où est en projet la construction d'une mosquée. Mais, en vertu de la loi de laïcité, il n'est pas du ressort de la municipalité de s'immiscer dans ce processus d'organisation, alors que dans le même temps cette intervention est réclamée par une partie des "pratiquants installés" voire par les nouvelles générations dans la mesure où elle apparaît comme un rempart à des ingérences extérieures ${ }^{12}$. Ce dilemme est amplifié à l'échelle nationale dans la perspective d'une réglementation de l'islam dans le cadre de la loi de 1905. Ceci renvoie au niveau d'intégration "systémique" de l'islam dans le cadre des institutions françaises qui doit être distingué du premier niveau précédemment décrit au sein duquel l'appartenance islamique fonctionne comme mode d'adaptation à l'environnement immédiat.

L'islam et la République

25 Jusqu'au début des années 1980, les gouvernants français ont eu tendance à considérer les dirigeants des principaux Etats d'origine comme les interlocuteurs privilégiés pour toute question afférente au statut de l'Islam, cette attitude consacrant la conception longtemps dominante d'une présence temporaire de ces populations, entrait par ailleurs en conjonction avec la position des Etats maghrébins. Le statut de la Grande Mosquée de Paris sous le contrôle de l'Algérie, apparaît comme le symbole de cette politique $^{13}$. Mais la multiplication des associations islamiques dans l'Hexagone va conduire à une réorientation du traitement institutionnel de l'islam et ce pour plusieurs raisons. D'une part, l'Etat ne peut se désintéresser du potentiel d'affrontements que suscite la demande d'islam dans les espaces locaux, demande bien souvent régulée en fonction du marché politique local, ce qui peut quelquefois conduire à des restrictions dans l'exercice du droit des cultes ${ }^{14}$. Ce souci de maintenir l'ordre social s'accompagne aussi d'une volonté de contrôler la multiplicité d'associations existantes au sein desquelles la parole produite pourrait constituer une infraction à l'ordre public ou dépendre d'influences extérieures portant atteinte à la souveraineté tatique. Ceci est d'autant plus irrecevable au regard des pouvoirs publics qu'une partie croissante des musulmans détient la nationalité française. Ces diverses raisons ont conduit en mars 1990 à la création sous l'égide de Pierre Joxe, alors ministre de l'Intérieur, du Conseil de Réflexion sur l'Islam en France (CORIF). Cette instance se composait de quinze membres représentant les principaux courants ethniques et nationaux de l'islam présents sur le territoire français et marquait l'ambition sousjacente de conduire à l'unification des populations musulmanes afin que le gouvernement puisse bénéficier d'un interlocuteur unique bien que Pierre Joxe ait pris soin de préciser qu'il n'était pas "Napoléon" et que la République ne déléguait aucune autorité aux quinze "sages" en matière d'organisation du culte, tout au plus les chargeait-elle de faire des propositions en vue de cette organisation ${ }^{15}$. Il n'en demeure pas moins que cette initiative conduit l'Etat à jouer un rôle dans le processus d'organisation de la minorité musulmane notamment en conférant aux membres du CORIF une légitimité supplémentaire dans la lutte en cours pour la représentativité 
dans le champ religieux. Avec le changement de majorité législative en mars 1993, le nouveau gouvernement a mis en sommeil le CORIF sans pour autant abandonner les négociations avec les principaux leaders musulmans. Toutefois, le rôle du recteur de la Grande Mosquée de Paris qui avait été minoré par la création du CORIF ${ }^{16}$, redevient central puisqu'avec le soutien du nouveau ministre de l'Intérieur, il a créé le 25 novembre 1993, un Conseil Consultatif des Musulmans de France regroupant les leaders associatifs les plus reconnus dans les principales régions ainsi que des fédérations d'associations comme l'Union des Organisations Islamiques de France (UOIF), l'Union des Etudiants Islamiques ou le Tabligh, association de réislamisation piétiste, implantée dans la plupart des pays européens où résident des populations musulmanes et plus connue en France sous le nom de "Foi et Pratique". Mais la majorité des membres du CORIF ainsi que la Fédération Nationale des Musulmans de France (FNMF) ont boudé cette initiative. L'objectif de ce nouveau Conseil est toujours le même: tenter le rassemblement des populations musulmanes mais cette fois autour de la Grande Mosquée de Paris et encore une fois, les diverses composantes de la populations musulmane marquent leurs réticences à ce projet puisqu'au même moment s'est constituée une initiative concurrente, le Conseil supérieur des Imams réunissant les responsables des principales mosquées de la région parisienne rivales de la Grande Mosquée ${ }^{17}$.

26 Ces diverses stratégies révèlent que la question de la représentativité des populations musulmanes est un enjeu éminemment politique pris en charge par des acteurs dont certains sont les relais des intérêts des Etats d'origine. Mais ceci a très peu de rapport avec la mise en forme juridique du culte qui lui ne pose pas plus de difficultés que celles afférant au judaïsme. En effet, les questions à résoudre font l'objet d'une jurisprudence abondante (abattage rituel, kashrout, aumônerie, régimes spéciaux dans les administrations ...) qui par analogie peut tout à fait être appliquée à l'islam en tant que culte. Ceci explique d'ailleurs que les travaux du CORIF soient demeurés d'une portée limitée : ils ont concerné symboliquement l'établissement d'une date commune pour le début du jeûne du mois de Ramadan et la réactualisation d'une circulaire de 1975 relative à aménagement de carrés musulmans dans les cimetières. Cependant, le problème le plus saillant, réfère au statut des imams et à leur formation dans la mesure où la plupart des autorités religieuses exerçant aujourd'hui dans l'Hexagone sont étrangères et ont été formées à l'étranger. Cette question a été débattue lors de la création d'une université islamique dans l'Ain en 1991 à l'aide de capitaux saoudiens et dont les étudiants comme les enseignants sont étrangers. L'inauguration le 17 novembre 1993, d'un Institut de formation des imams sous l'égide de la Grande Mosquée et avec l'aval du ministre de l'Intérieur apparaît comme une réponse concurrente de cette première tentative et s'inscrit dans une perspective de gallicanisation des futures autorités religieuses ${ }^{18}$.

De manière plus fondamentale, il apparaît que le dialogue entre les pouvoirs publics et des leaders associatifs islamiques a pour objectif de faire ratifier par les musulmans le pacte laïque républicain tout en négociant avec eux un contrat séparé. Mais ceci pose la question de la dissociation entre exercice du culte et dimension sociale de la religion, laquelle est au fondement de ce pacte mais ne correspond pas véritablement aux modalités d'identification à l'islam de la plupart des "pratiquants installés" surtout dans un moment où d'autres minorités religieuses remettent en cause cette dissociation ${ }^{19}$. En d'autres termes, les références ethniques ou culturelles contribuent davantage à définir la relation à l'islam des musulmans que la référence canonique aux 
cinq piliers du culte. Ceci explique d'ailleurs les difficultés de mise en place d'un consensus sur autorité religieuse la plus habilitée à négocier avec les pouvoirs publics.

En définitive, cet écart entre les attentes politiques d'une part et les demandes sociales d'autre part constaté à propos des relations entre l'islam et l'Etat doit également être replacé dans un contexte plus large de remise en cause de la dissociation institutionnelle qui constitue l'un des aspects fondamentaux de la laïcité. En effet, la laïcité de l'Etat ne signifie pas seulement que celui-ci se situe désormais en dehors de toute obédience religieuse, ce qui était un aspect déjà connu sous le Concordat. Elle implique aussi le refus de régime de droit public à toute forme d'activité religieuse, ce qui conduit à la privatisation de la religion et donc au déclin de sa légitimité sociale ${ }^{20}$. L'esprit de la loi de 1905 produit donc une coupure entre exercice du culte et la dimension sociale voire collective de la religion. Ceci va conduire à façonner les représentations sociales qui sous-tendent le principe de laïcité et qui confèrent à rendre illégitimes toute expression publique de l'appartenance religieuse. Preuve en est, la passion soulevée par l'entrée de foulards islamiques dans les écoles publiques en novembre 1989, qui a conduit le Conseil d'Etat à rendre un avis dans lequel il était rappelé que la loi de laïcité implique la neutralité des agents du service public et non pas celle des usagers dans la limite de leur respect de l'ordre public ${ }^{21}$. Cette méfiance envers l'expression publique de l'appartenance religieuse met en évidence les résistances ${ }^{22}$ à repousser une frontière entre espace public et privé qui semblait définitivement consacrée depuis 1905 par la pratique sociale, mais qui ne parait plus adaptée aujourd'hui non seulement aux nouveaux venus que sont les musulmans, mais aussi à l'ensemble des groupes religieux.

\section{NOTES}

1. Les représentations collectives relatives à l'islam ont en effet été façonnées par l'histoire coloniale. Il ne faut pas oublier qu'à cette période, si les musulmans en Algérie étaient des sujets français, ils ne pouvaient devenir citoyens qu'à la condition de renoncer aux règles de Statut personnel.

2. Cette analyse est le résultat d'une enquête qui s'est déroulée entre 1987 et 1991 après des populations musulmanes installées en France et qui a fait l'objet d'une thèse. Cf. J. Césari, Etre musulman en France. Associations, militants et mosquées, Paris, Ed. Karthala, 1994.

3. A cet égard, il aura fallu près d'un demi-siècle de noria ininterrompue pour que l'émigration de travail algérienne se transforme définitivement en émigration de peuplement. Voir Belkacem Hifi, L'immigration algérienne en France. Origines et perspectives de non retour. Paris, Ed. L'Harmattan/CIEM, 1985, p. 145 et suivantes. De même, l'adhésion syndicale, notamment à la CGT durant la période d'activité est attestée mais elle n'a pas eu pour conséquence une politisation équivalente à celle qui s'est produite dans d'autres 
migrations telles que l'italienne ou polonaise. Voir Maryse Tripier, L'immigration dans la classe ouvrière en France. Thèse d'Etat. Nantes, Université de Nantes, 1987.

4. Pour ces générations, le défi est le suivant : comment construire une carte cognitive cohérente alors qu'elles sont prises entre un système économique et politique conçu comme plus efficace et plus gratifiant que celui des pères et les bribes d'un système culturel dont les valeurs perdurent mais dont les prescriptions sont de moins en moins pertinentes ? Ceci conduit à une mise en cohérence de ces valeurs différentes où la "valorisation du pragmatique" (accès aux droits civiques et sociaux, recherche de gratifications matérielles) coexiste avec le maintien d'un minimum ontologique (valeurs liées à l'arabité et à l'Islam transmises par la famille). Il en résulte que pour la majorité d'entre eux et l'identification à l'islam est envisagée comme source de valeurs donnant du sens à leur vie sans pour autant impliquer chez eux un passage à la pratique. Ce processus n'est pas sans rappeler les nouvelles formes de religiosité dans les sociétés modernes où le croyant n'obéit plus aux normes transmises par l'institution ou la tradition mais choisit "les biens du salut" en fonction de son individualité. Cf. Françoise Champion et Danièle Hervieu-Léger (dir.), De l'émotion en religion. Renaissance et traditions, Paris, Le Centurion, 1990.

5. Beaucoup invoquent d'ailleurs un Hadith du Prophète selon lequel lorsque le croyant est en voyage, il n'est pas astreint au respect strict des prescriptions.

6. A ce jour, on recense près de 1000 associations islamiques déclarées gérant des lieux de culte, la quasi totalité relevant du régime associatif loi de 1901.

7. Il convient de préciser que cette "manne" financière n'est pas aussi abondante que l'on pourrait le croire à l'exception de quelques cas où les promoteurs de projets de mosquées sont bien insérés dans des réseaux transnationaux leur permettant de cumuler des sources de financements plurielles provenant de divers lieux du monde arabomusulman. Il n'est que de constater le faible nombre de mosquées construites sur le territoire en une décennie (Evry, Lyon, Mantes-laJolie). De plus, ces aides financières ont eu tendance à diminuer après la guerre du Golfe. Cf. Césari (J.), op. cit. (sur les péripéties du projet de mosquée à Marseille, chapitre VI : "Une mosquée-cathédrale", pp. 111-126). Le terme de communauté ne peut être employé qu'en tenant compte de cette double logique : d'une part, la reconstitution et réactivation des réseaux de solidarité liés à l'origine ethnique et nationale, d'autre part le fonctionnement de ces réseaux à l'échelle d'un quartier.

8. Cette distinction citoyenneté passive/citoyenneté négative est empruntée à Jean Leca : "Individualisme et citoyenneté" in Jean Leca et Pierre Birnbaum (dir.), Sur l'individualisme, Paris, Presses de la FNSP, 1991, pp. 159-209.

9. Comme l'énonce Camille Mansour : "Il s'agit de la plus belle réalisation de l'idéologie musulmane : contraindre le pouvoir, tous les 
pouvoirs à reconnaître au moins verbalement l'Islam, à investir les quadis, à faire appliquer la Shari'a. Mais à part cela, le pouvoir reste jaloux de sa sphère propre : les décisions politiques. Tout se passe donc comme s'il y avait dans l'Etat musulman une séparation de l'Islam et de l'Etat, ou plutôt de l'Islam plus la Shari's (surtout le droit privé) et de l'Etat moins le droit privé" in : L'autorité dans la pensée musulmane. Paris, Ed Jean Vrin, 1975, p. 93.

10. Sur cet impensé de la condition minoritaire voir Bernard Lewis : "La situation des populations musulmanes dans un régime non musulman" in Dominique Schapper et Bernard Lewis (dir.), Musulmans en Europe, Arles, Actes Sud, 1992, pp. 11-34.

11. Tout d'abord parce qu'elle ne répond pas à ce jour aux attentes de la majorité des nouvelles générations nées sur le territoire français et que par ailleurs elle ne s'inscrit pas dans les objectifs immédiats des pratiquants qui construisent aujourd'hui l'islam de France. A cet égard, la demande de mosquées, d'écoles coraniques ou de carrés musulmans dans les cimetières apparaissent davantage comme des enjeux prioritaires.

12. C'est ainsi que certains ont pu se déclarer favorables au financement des mosquées par les collectivités locales ou l'Etat. Cf. Césari (J.), op. cit.

13. A propos de l'historique de cette mosquée voir Gilles Kepel, Les banlieues de l'Islam, Paris, Ed. Seuil, 1987. La nomination du Recteur de cette institution est laissée à l'appréciation du pouvoir algérien. Ceci a d'ailleurs conduit en 1992 à une crise à la tête de cette instance, directement liée à la mise en place d'un Haut Comité d'Etat en Algérie après la suspension du processus électoral. En effet, Tidjani Heddam, alors Recteur de la grande mosquée a été nommé membre de ce Haut Comité, ce qui a entraîné une polémique avec les autorités françaises à propos de son maintien dans la fonction de recteur. Les dirigeants algériens s'étant laissés convaincre de l'impossibilité du cumul des mandants, ont finalement accepté le 12 avril 1992, la nomination de Dalil Boubakeur, qui est ainsi devenu le premier recteur de nationalité française.

14. En effet, un projet de mosquée suscite des réactions diverses de la part des élites locales pouvant aller de la résistance sourde à l'hostilité déclarée voire au déni du libre exercice des cultes comme à CharvieuChavagneux au cours de l'été 1989 ou encore à Libercourt en avril 1991.

15. Cf. entretien accordé au Monde le 17 mars 1989.

16. La création du CORIF avait également comme objectif de battre en brèche les différentes tentatives d'hégémonie sur l'Islam de France par les Recteurs successifs.

17. Le Monde, 3 décembre 1993. Le 10 janvier 1995, le recteur Dalil Boubakeur a solennellement remis à Charles Pasqua une "Charte des musulmans de France" voulant concrétiser l'institutionnalisation de "l'islam de France" : la cérémonie a été fortement critiquée par de grandes associations, dont l'UOIF (Le Monde, 12 janvier 1995). 
18. Il faut également mentionner un autre lieu de formation pour imams, cette fois sous l'égide de la Fédération nationale des musulmans de France (FNMF) à Mantes-la-Jolie qui a ouvert ses portes au mois d'octobre 1993.

19. Il n'est que de constater la prise de parole de plus en plus affirmée des clercs de l'ensemble des religions sur une série d'enjeux sociaux (immigration, port du foulard, etc.) ou leur entrée dans des instances telles que le Comité national d'éthique pour les sciences de la vie et de la santé ou le Conseil national sur le Sida.

20. Il faut quand même préciser que ce principe a été assoupli notamment avec l'émergence d'une distinction entre la confession et l'action sociale qui a permis le financement par les pouvoirs publics d'activités éducatives gérées par des institutions religieuses, mais aussi médicales, caritatives, etc.

21. Avis du 27 novembre 1989.

22. La circulaire Bayrou sur le port de signes religieux ostentatoires dans l'école publique est venue relancer la polémique en septembre 1994 et a conduit à l'exclusion de collégiennes "portant foulard" dans un certain nombre d'établissements. La légalité de cette circulaire est en cours d'examen, et le Conseil d'Etat sera sans doute amené à se prononcer sur sa validité (Le Monde, 20 décembre 1994). 